\title{
A Generalization of the Kac-Moody Algebras with a Parameter on an Algebraic Curve and Perturbations of Solitons
}

\author{
V. G. Mikhalev \\ Department of Computer Sciences, Vladimir State Teachers Institute, Prospect Stroiteley II, \\ SU-600024 Vladimir, USSR
}

Received April 10, 1990

\begin{abstract}
The Lie-algebraic approach for the dynamic systems associated with a generalization of the Kac-Moody algebras on Riemann surfaces is developed. A technique of solving the inverse scattering problem of operators with spectral parameters on Riemann surfaces is presented. Some equations associated with generalized Kac-Moody algebras are presented. The connection between their hamiltonian structure and deformed Lax representation is discussed as well as its applications to some special perturbations of integrable systems.
\end{abstract}

\section{Introduction}

The Lie-algebraic approach to the theory of completely integrable nonlinear equations makes it possible to connect a classical $r$-matrix formalism, representations of zero curvature and Poisson brackets of the coefficients of transition matrices. Kostant [1] was the first who proposed the method of construction of integrable systems by dividing a Lie algebra to a direct sum of two subalgebras $\mathfrak{5}=\mathfrak{G}_{+} \oplus \mathfrak{b}_{-}$. Then in works of the Leningrad-school researchers (see [2] and reference in it) this method was adapted to a wide class of the Lie algebras including infinite dimensional ones. Almost all known nonlinear equations admitting the Lax representation were plunged into this approach.

In this paper the same approach is developed for some Lie algebras dividing as

$$
\mathfrak{6}=\mathfrak{b}_{+} \oplus \mathfrak{b}_{0} \oplus \mathfrak{G}_{-},
$$

where $\mathfrak{G}_{ \pm}$are subalgebras. $\mathfrak{b}_{0}$ is a finite dimensional subspace. They are used in consideration of the operator bundles parametrized by point on an algebraic curve of genus $g>0$. For a class of such operators the Gelfand-Lefitan-Marchenko approach to the solution of the inverse scattering problem was developed in [3]. In Sect. 3 its modified version will be given without proofs. As it follows from [4], the algebra of those operators inevitably includes $\mathfrak{G}_{0}$. It will be seen that most aspects of the Lie-algebraic approach require generalizations. 
Instead of classical $r$-matrix constructed by the operator

$$
\hat{R}=\frac{1}{2} \hat{P}_{+}-\frac{1}{2} \hat{P}_{-},
$$

one must consider two operators

$$
\widehat{R}=\frac{1}{2} \widehat{P}_{+}-\frac{1}{2} \widehat{P}_{-}, \quad \hat{L}=\hat{P}_{0},
$$

where $\hat{P}_{ \pm}, \hat{P}_{0}$ are projectors on $\mathfrak{G}_{ \pm}, \mathfrak{G}_{0}$. The operators $\hat{R}$ and $\hat{L}$ satisfy a system of equations generalizing the modified classical Yang-Baxter equation [5].

The nonlinear equations associated with such algebras are not completely integrable ones. They admit a representation more general than the Lax one.

The Poisson brackets of the transition matrix coefficients can be calculated by the corresponding generalization of the classical $r$-matrix.

In conclusion I shall put some words about applications of constructions mentioned below to those perturbations of integrable systems which result not only in small deformations of invariant tori but also in their bifurcations.

\section{Generalization of the Lie-Algebraic Approach in the Case of Operators Bundle with a Parameter on an Algebraic Curve of Genus $g>0$}

Suppose that the Lie algebra $\mathfrak{5}$ as a linear space may be broken up

$$
\mathfrak{G}=\mathfrak{G}_{+} \oplus \mathfrak{G}_{0} \oplus \mathfrak{G}_{-},
$$

where $\mathfrak{G}_{0}$ is a finite dimensional subspace. Let us consider at the same time an ordinary commutator in $\mathfrak{G}$ and also a new one

$$
\begin{aligned}
{[\xi, \eta]_{0}=} & {\left[\hat{P}_{+} \xi, \hat{P}_{+} \eta\right]-\left[\hat{P}_{-} \xi, \hat{P}_{-} \eta\right] } \\
= & {\left[\hat{R}_{\xi}, \eta\right]+[\xi, \hat{R} \eta]-[\hat{R} \xi, \hat{L} \eta]-[\hat{L} \xi, \hat{R} \eta], } \\
& \quad \hat{R}=\frac{1}{2} \hat{P}_{+}-\frac{1}{2} \hat{P}_{-}, \quad \hat{L}=\hat{P}_{0} .
\end{aligned}
$$

The commutator (1) also defines the Poisson-Kirillov bracket

$$
\{\varphi, \psi\}_{0}=\left\langle[\nabla \varphi, \nabla \psi]_{0}, u\right\rangle, \quad \varphi, \psi \in \mathfrak{G}^{*} .
$$

\section{Derivation of the Equations Generalizing the Yang-Baxter Ones}

Write down the Jacoby identity for (1). After manipulations we obtain

$$
\begin{aligned}
& {\left[\xi,[\eta, v]_{0}\right]_{0}+\text { c.c. }=} {[(1-L) \xi, B(\eta, v)]+[(1-L) \eta, B(v, \xi)]+[(1-\hat{L}) v, B(\xi, \eta)] } \\
&+[\hat{R} \xi, C(\eta, v)]+[\hat{R} \eta, C(v, \xi)]+[\hat{R} v, C(\xi, \eta)], \\
& B(\xi, \zeta)=\hat{R}[\hat{R} \xi,(1-\hat{L}) \eta]+R[(1-\hat{L}) \xi, \hat{R} \eta]-[\hat{R} \xi, \hat{R} \eta], \\
& C(\xi, \eta)=\hat{L}(\hat{R} \xi,(1-\hat{L}) \eta]+\hat{L}[(1-\hat{L}) \xi, \hat{R} \eta] .
\end{aligned}
$$

Theorem 1. (1) and (2) admit the Jacoby identity, if $\hat{R}$ and $\hat{L}$ satisfy the following system of equations generalizing the modified classical Yang-Baxter equation

$$
\begin{gathered}
B(\xi, \eta)=\alpha[(1-\hat{L}) \xi,(1-\hat{L}) \eta], \\
C(\xi, \eta)=\beta[\hat{R} \xi, \hat{R} \eta] .
\end{gathered}
$$


Consequence. (1) and (2) admit the Jacoby identity if $\mathbf{6}_{ \pm}$are subalgebras.

The proof may be performed by the direct computation.

\section{The Generalization of the Kac-Moody Algebras to the Riemann Surfaces of Genus $\mathrm{g}>0$}

In this subsection necessary for future information from [4] is presented.

Let $\Gamma$ be a nonspecific algebraic curve of genus $g$ with two groups of fixed points $P_{i}^{ \pm} . \mathscr{A}$ denotes the space of meromorphic functions with poles lying only at $P_{i}^{ \pm}$. It has a natural ring structure. Its additive basis was constructed in [4]. The dual space $\mathscr{A}^{*}$ is formed by 1 -forms. A coupling given by integration along a contour $C$ from the set was suggested in [4]. The dual basis is generated by forms $\omega_{i}$ such that

$$
\oint_{C} A_{i} \omega^{j}=\delta_{i}^{j} .
$$

A 1 -form $\omega_{0}$ makes a bilinear mapping $\mathscr{A} \otimes \mathscr{A} \rightarrow \mathbb{C}$

$$
C^{\omega_{0}}\left(A_{i}, A_{j}\right)=\oint_{C} A_{i} A_{j} \omega_{0}
$$

Any operator $\hat{R} \in$ End $\mathscr{A}$ is defined by the kernel $R(\lambda, \mu)$ which is 1 -form with respect to $\mu$ and 0 -form with respect to $\lambda$, so that

$$
\hat{R} A(\lambda)=\oint_{C_{\mu}} R(\lambda, \mu) A(\mu) .
$$

The algebra $\mathscr{A}$ can be divided

$$
\mathscr{A}=\mathscr{A}_{+} \oplus \mathscr{A}_{0} \oplus \mathscr{A}_{-},
$$

where $\mathscr{A}_{+}, \mathscr{A}_{-}$are subalgebras of functions which poles are only at $P_{i}^{+}, P_{i}^{-}$ correspondingly; $\mathscr{A}_{0}$ is a finite dimensional addition of $\mathscr{A}_{+} \oplus \mathscr{A}_{-}$to the algebra.

Example. Let us consider the algebraic curve of genus 1 defined in $\mathbb{C}^{3}$ by the relations

$$
w_{1}^{2}=1-\frac{\alpha_{1}}{4 \lambda^{2}}, \quad w_{2}^{2}=1-\frac{\alpha_{2}}{4 \lambda^{2}} .
$$

The ring $\mathscr{A}$ consist of meromorphic functions with poles only at 8 points $\lambda=\infty$ and $\lambda=0$. The basis in $\mathscr{A}$ is generated by elements of view

$$
A_{i}^{0}=\lambda^{i}, \quad A_{i}^{1}=w_{1} \lambda^{i}, \quad A_{i}^{2}=w_{2} \lambda^{i}, \quad A_{i}^{3}=w_{1} w_{2} \lambda^{i} .
$$

The dual space is determined in regard to coupling defined by integration along the contour $C=\{|\lambda|=1\}$, oriented anticlockwise. The dual basis is generated by 1 -forms

$$
\omega_{0}^{i}=\frac{\lambda^{-i-1} d \lambda}{8 \pi i}, \quad \omega_{1}^{i}=\frac{\lambda^{-i-1} d \lambda}{8 \pi i \omega_{1}}, \quad \omega_{2}^{i}=\frac{\lambda^{-i-1} d \lambda}{8 \pi i w_{2}}, \quad \omega_{3}^{i}=\frac{\lambda^{-i-1} d \lambda}{8 \pi i w_{1} w_{2}} .
$$

The algebra $\mathscr{A}$ includes two subalgebras

$$
\begin{gathered}
\mathscr{A}_{+}=\left\{A_{i}^{0}, A_{j}^{1}, A_{j}^{2}, A_{k}^{3}, i \geqq 0, j \geqq 1, k \geqq 2\right\}, \\
\mathscr{A}_{-}=\left\{A_{i}^{0}, A_{j}^{1}, A_{j}^{2}, A_{j}^{3}, i<0, j \leqq 0\right\},
\end{gathered}
$$


and an addition $\mathscr{A}_{0}=\left\{A_{1}^{3}\right\}$. It is necessary for applications to the theory of nonlinear equations to extend $\mathscr{A}_{0}$ for account of $\mathscr{A}_{+}$and $\mathscr{A}_{-}$. In particular $I^{\prime} 11$ consider a case with

$$
\mathscr{A}_{0}=\left\{A_{0}^{0}, A_{0}^{1}, A_{0}^{2}, A_{0}^{3}, A_{1}^{3}\right\} \text {. }
$$

A unity operator in $\mathscr{A}$ is determined by the kernel

$$
\delta_{C}(\tilde{\lambda}, \lambda)=\left(1+\frac{\tilde{w}_{1}}{w_{1}}+\frac{\tilde{w}_{2}}{w_{2}}+\frac{\tilde{w}_{1} \tilde{w}_{2}}{w_{1} w_{2}}\right) \frac{1}{4} \delta(\lambda-\tilde{\lambda}) d \lambda
$$

and projectors $\hat{P}_{0}, \hat{P}_{ \pm}$by

$$
\begin{gathered}
P_{0}(\tilde{\lambda}, \lambda)=\left(1+\frac{\tilde{w}_{1}}{w_{1}}+\frac{\tilde{w}_{2}}{w_{2}}+\frac{\tilde{w}_{1} \tilde{w}_{2}}{w_{1} w_{2}}\left(1+\frac{\tilde{\lambda}}{\lambda}\right)\right) \frac{d \lambda}{8 \pi i \lambda}, \\
P_{-}(\tilde{\lambda}, \lambda)=\frac{1}{2} \delta_{C}(\tilde{\lambda}, \lambda)+\left(1+\frac{\tilde{w}_{1}}{w_{1}}+\frac{\tilde{w}_{2}}{w_{2}}+\frac{\tilde{w}_{1} \tilde{w}_{2}}{w_{1} w_{2}}\right) \text { V.p. } \frac{d \lambda}{8 \pi i(\tilde{\lambda}-\lambda)}, \\
P_{+}(\tilde{\lambda}, \lambda)=\delta_{C}(\tilde{\lambda}, \lambda)-P_{0}(\tilde{\lambda}, \lambda)-P_{-}(\tilde{\lambda}, \lambda) .
\end{gathered}
$$

The multiplicative structure of $\mathscr{A}$ results in our being able to determine for any semi-simple matrix algebra an algebra

$$
\mathfrak{G}=\operatorname{Mat} \otimes \mathscr{A}
$$

which is a generalization of the Kac-Moody one to the case of arbitrary Riemann surfaces of genus $g>0$. That algebra consists of matrices, which elements belong to the ring $\mathscr{A}$. The dual space consists of matrices, which elements are $I$-forms. Bilinear coupling is defined by the expression

$$
(\xi, \eta)=\frac{1}{\operatorname{dim} \mathrm{Mat}} \operatorname{Sp}_{C} \xi \eta, \quad \xi \in \mathfrak{G}, \eta \in \mathfrak{G}^{*} .
$$

Let $\tilde{\mathfrak{G}}=C^{\infty}\left(S^{1}, \mathfrak{5}\right)$ denote an algebra of periodic infinite differentiable with respect to $x$ functions taking values in $\mathfrak{G}^{\text {. Let }}$ us construct its central extension $\tilde{\mathfrak{G}}^{\wedge}$ by the generalization of the Maurer-Cartan cocycle

$$
\gamma^{\omega_{0}}(\xi, \eta)=\frac{1}{\operatorname{dimMat}} \operatorname{Sp} \int_{0}^{2 \pi} d x \oint_{C_{\lambda}} \xi(x, \lambda) \partial_{x} \eta(x, \lambda) \omega_{0}(\lambda)
$$

\section{Hamiltonian Structure of $\left(\tilde{\mathfrak{G}}^{\wedge}\right)^{*}$}

The decomposition (3) induces the following one:

$$
\tilde{\mathfrak{G}}=\tilde{\mathfrak{G}}_{+} \oplus \tilde{\mathfrak{G}}_{0} \oplus \tilde{\mathfrak{F}}_{-},
$$

where $\tilde{\mathfrak{F}}_{ \pm}$are subalgebras too. Therefore, in accordance with the consequence of Theorem 1, the commutator (1) and the bracket (2) also admit the Jacoby identity. The bracket (2) can be rewritten in the form

$$
\begin{aligned}
&\{U(x, \lambda) \otimes U(y, \mu)\}_{0} \\
&=\delta(x-y) R(\lambda, \mu)[\Pi, U(x, \lambda) \otimes 1]-R(\mu, \lambda)[\Pi, 1 \otimes U(x, \mu)]) \\
&+\delta^{\prime}(x-y) \Pi\left(R(\lambda, \mu) \omega_{0}(\lambda)+R(\mu, \lambda) \omega_{0}(\mu)\right) \\
&-\oint_{C_{\lambda}}(R(v, \lambda) L(v, \mu)+R(v, \mu) L(v, \lambda) \\
& \times\left([\Pi, U(x, v) \otimes 1] \delta(x-y)+\Pi \omega_{0}(v) \delta^{\prime}(x-y)\right),
\end{aligned}
$$


where $\Pi=\sigma^{i} \otimes \sigma_{i}, \sigma_{i}$ - form an orthonormal basis in $\mathfrak{G}, U(x, \lambda) \in\left(\widetilde{\mathfrak{G}}^{\wedge}\right)^{*}, R(\lambda, \mu)$, $L(\lambda, \mu)$ are kernels of operators $\hat{R}$ and $\hat{L}$.

In applications (6) should be reduced to an invariant finite-dimensional submanifold. The reduction may be done by choice of appropriate $\mathfrak{6}_{0}$ and $\omega_{0}$ because the width of $\mathfrak{G}$ grading is limited.

I shall imply below that $U(x, \lambda)$ belongs to such an invariant submanifold $M \in\left(\widetilde{\tilde{F}}^{\wedge}\right)^{*}$. Let me introduce a fundamental solution $T(x, y, \lambda)$,

$$
\omega_{0}(\lambda) T_{x}(x, y, \lambda)=U(x, \lambda) T(x, y, \lambda), \quad T(y, y, \lambda)=1,
$$

and a transition matrix $T(2 \pi, 0, \lambda)$.

As in the polynomial bundle case the following theorem is proved by direct computation

Theorem 2. The invariants of coadjoint action have the form

so as

$$
\oint \operatorname{Sp} T^{n}(2 \pi, 0, \lambda) \omega(\lambda),
$$

$$
\oint_{C_{\mu}}\left\{U(x, \lambda), \operatorname{Sp} T^{n}(2 \pi, 0, \mu)\right\} \omega(\lambda)=0 .
$$

It should be noted that because of $\mathfrak{F}_{0}$ the invariants of coadjoint action shall not be commuted with respect to (2). Using (6) and Theorem 2 the bracket

$$
\{U(x, \lambda), F\}_{0}, \quad F=\oint_{C_{\mu}} \operatorname{Sp} T^{n}(2 \pi, 0, \mu) \omega(\mu)
$$

can be presented in the form

$$
\begin{gathered}
\{U(x, \lambda), F\}_{0}=\left(1-\hat{P}_{0}^{*}\right)\left(\left[V_{1}(x, \lambda), U(x, \lambda)\right]+V_{1 x}(x, \lambda) \omega_{0}(\lambda)\right) \\
+\hat{P}_{-}^{*}\left(\left[V_{2}(x, \lambda), U(x, \lambda)\right]+V_{2 x}(x, \lambda) \omega_{0}(\lambda)\right), \\
V_{1}(x, \lambda)=n \cdot \operatorname{dim} M \cdot \hat{P}_{+}\left(\frac{\omega(\lambda)}{\omega_{0}(\lambda)} T(x, 0, \lambda) T^{n-1}(2 \pi, 0, \lambda) T(2 \pi, x, \lambda)\right), \\
V_{2}(x, \lambda)=n \cdot \operatorname{dim} \mathrm{Mat} \cdot \hat{P}_{0}\left(\frac{\omega(\lambda)}{\omega_{0}(\lambda)} T(x, 0, \lambda) T^{n-1}(2 \pi, 0, \lambda) T(2 \pi, x, \lambda)\right) .
\end{gathered}
$$

Here the star denotes an adjoint operator defined by the same kernel. The relation (7) is a generalized Lax representation. This statement is in agreement with the result of [8] that in the general case there are no ordinary Lax representations parametrized by point on an algebraic curve of genus $g>0$. I shall restrict myself by the case when, after a specific choice of the Riemann surface, $(7)$ can be written in the form

$$
L_{t}=[L A]+\varepsilon B
$$

with some $B$, where $\varepsilon$ is a small variable.

If $U(x, t, \lambda)$ evolved due to the equation

$$
U_{t}(x, t, \lambda)=\{U(x, t, \lambda), F\}
$$

the dynamic of the transition matrix is determined, taking into account $\left(7^{\prime}\right)$, by the following equation:

$$
\begin{aligned}
T_{t}(2 \pi, 0, t, \lambda)= & A(2 \pi, \lambda, t) T(2 \pi, 0, t, \lambda)-T(2 \pi, 0, t, \lambda) A(0, \lambda, t) \\
& +\varepsilon \int_{0}^{2 \pi} T(2 \pi, x, t, \lambda) B(x, t, \lambda) T(x, 0, t, \lambda) d x
\end{aligned}
$$


Let me present the result of the calculations of Poisson brackets of transition matrix coefficients

$$
\{T(\lambda) \otimes T(\mu)\}_{0}^{\wedge}=[r(\lambda, \mu), T(\lambda) \otimes T(\mu)]+\frac{\Omega(\lambda, \mu)}{\omega_{0}(\lambda) \omega_{0}(\mu)},
$$

where

$$
r(\lambda, \mu)=\frac{1}{2} \Pi\left(\frac{R(\lambda, \mu)}{\omega_{0}(\mu)}-\frac{R(\mu, \lambda)}{\omega_{0}(\lambda)}\right)
$$

and the form $\Omega(\lambda, \mu)$ is determined by the relation

$$
\begin{aligned}
\Omega(\lambda, \mu)= & \int_{0}^{2 \pi} d x \oint_{C_{v}}(R(v, \lambda) L(v, \mu)+R(v, \mu) L(v, \lambda)) \\
& \times(T(2 \pi, x, \lambda) \otimes T(2 \pi, x, \mu)[\Pi, 1 \otimes U(x, v)] T(x, 0, \lambda) \otimes T(x, 0, \mu) \\
& +T(2 \pi, x, \lambda) \otimes T_{x}(2 \pi, x, \mu) \Pi T(x, 0, \lambda) \otimes T(x, 0, \mu) \omega_{0}(v) \\
& \left.+T(2 \pi, x, \lambda) \otimes T(2 \pi, x, \mu) \Pi T(x, 0, \lambda) \otimes T_{x}(x, 0, \mu) \omega_{0}(v)\right) .
\end{aligned}
$$

\section{The Investigation of Operators with a Parameter} on an Algebraic Curve by Inverse Scattering Transform

Let us consider an operator $L$ of the form

$$
\begin{gathered}
L=\partial_{x}-U, \\
U=i \sigma_{3} \lambda+\sum_{i=1}^{3} \sum_{j=0}^{N_{i}} \sum_{k=0}^{n-1} \varphi_{i j k}(x) \lambda^{j} \omega^{k} \sigma_{i},
\end{gathered}
$$

where $\sigma_{i}$ are the Pauly matrices, $\lambda$ and $\omega$ are connected by an algebraic relation of view

$$
\omega^{n}=\sum_{\ell=1}^{n} P_{\ell}(\lambda) \omega^{n-\ell}
$$

$P_{\ell}(\lambda)=\sum_{m} P_{\ell}^{m} \lambda^{m}$ are polynomials of $\lambda$ which highest power $\leqq M, \varphi_{i j k}(x)$ are infinite differentiable functions of $x$.

Equation (11) gives an algebraic curve $\Gamma$ which may be seen as $n$-sheets covering over $\lambda$-plane. The monomials $\lambda^{j} \omega^{k}, 0 \leqq k \leqq n-1$ form a basis in $\mathscr{A}-$ the space of meromorphic functions having poles lying only at $2 n$ points $P_{i}^{ \pm}$: $P_{i}^{+}=\{\lambda=\infty\}, P_{i}^{-}=\{\lambda=0\}$. The elements $\lambda^{j} \omega^{k} \sigma_{i}$ form a basis in the algebra $\operatorname{sl}(2, \mathscr{A})$ which is the analog of the Kac-Moody algebra with a parameter on an algebraic curve. Thus, $U \in \operatorname{sl}(2, \mathscr{A}, \mathbb{R})$ is the algebra of infinite differentiable functions taking their values in $\operatorname{sl}(2, \mathscr{A})$.

We can introduce a general grading in $\mathscr{A}$ through the powers of $\lambda$,

$$
\begin{gathered}
\mathscr{A}=\stackrel{\oplus}{j=-\infty}^{+\infty} \mathscr{A}_{j}, \quad \mathscr{A}_{j}=\left\{\lambda^{j}, \omega \lambda^{j}, \ldots, \omega^{n-1} \lambda^{j}\right\}, \\
g \in \mathscr{A}_{j}, \quad h \in \mathscr{A}_{j}, \quad g h \in \bigoplus_{k=i+j}^{i+j+q} \mathscr{A}_{k},
\end{gathered}
$$


the width of grading $q$ is finite and obeys the estimation $q \leqq M^{n-1}$.

Let us demand that $\varphi_{i j k}(x) \underset{|x| \rightarrow \infty}{\underset{1}{3}} 0$ more rapid than $x$ to any finite power and hence are the Schwartz functions. Now turn to the study of the spectral theory of the $L$-operator with rapidly decreasing boundary conditions. We must consider a fundamental solution

$$
L(x, \lambda, \omega) T(x, y, \lambda, \omega)=0, \quad T(y, y, \lambda, \omega)=1,
$$

Jost functions

$$
\begin{gathered}
\psi_{-}(x, \lambda, \omega)=\lim _{y \rightarrow-\infty} T(x, y, \lambda, \omega) e^{-i \lambda \sigma_{3} y}, \\
\psi_{+}(x, \lambda, \omega)=\lim _{y \rightarrow+\infty} T(x, y, \lambda, \omega) e^{-i \lambda \sigma_{3} y}, \\
\lim _{y \rightarrow-\infty}\left(\psi_{-}(x, \lambda, \omega)-e^{i \lambda \sigma_{3} x}\right)=0, \\
\lim _{y \rightarrow-\infty}\left(\psi_{+}(x, \lambda, \omega)-e^{i \lambda \sigma_{3} x}\right)=0,
\end{gathered}
$$

and transition matrix $T, \psi_{+} T=\psi_{-}$.

Elsewhere the terms " $\lambda$-half-planes" or " $\lambda$-axes" will mean connected pieces of the inverse image on $\Gamma$ of $\lambda$ belonging to corresponding half-plane or axis.

Theorem 3. The first column of $\psi_{+} e^{-i \lambda \sigma_{3} x}$ and the second column of $\psi_{-} e^{-i \lambda \sigma_{3} x}$ are analytical functions at the upper $\lambda$-half-planes of $\Gamma$; the second column of $\psi_{+} e^{-i \lambda \sigma_{3} x}$ and the first column of $\psi_{-} e^{-i \lambda \sigma_{3} x}$ are analytical functions at the under $\lambda$-half-planes of $\Gamma$.

As for the transition matrix

$$
T=\left(\begin{array}{ll}
d & b \\
c & a
\end{array}\right)
$$

the coefficient $a$ is an analytical function at the upper - half-planes of $\Gamma$ and $d$-at the under ones.

$b$ and $c$ are determined at real $\lambda$-axes where the relation $a d-b c=1$ is valid. Assume, that as in rational cases $b(\lambda) \underset{\lambda \rightarrow \infty}{\rightarrow} 0$ if $\varphi_{i j k}(x)$ are of Schwartz type.

For imaginary $\varphi_{i j k}(x)$ we have

$$
d(\lambda, \omega)=\bar{a}(\bar{\lambda}, \bar{\omega}), \quad c(\lambda, \omega)=-\bar{b}(\bar{\lambda}, \bar{\omega}) .
$$

If $\bar{\omega}=\omega, \operatorname{Im} \lambda=0$ then we obtain at the real $\lambda$-axes

$$
|a|^{2}+|b|^{2}=1 \text {. }
$$

Suppose that there are no branch points at real $\lambda$-axes. The inverse image of real $\lambda$-axis cut the Riemann surface $\Gamma$ on two connected pieces. In that case $a(\lambda)$ is completely determined at the upper $\lambda$-half-planes by its own zeroes and by the function $b(\lambda)$ determined at the real axes. For the aim the Gauchy-type kernel is necessary according to a contour which is an inverse image of the axis of real $\lambda$. So we have

$$
a(\lambda, \omega)=F(\lambda, \omega) e^{\frac{1}{2} \int_{-\infty, \Gamma}^{+\infty} K(\lambda, \omega, \tilde{\lambda}, \tilde{\omega}) \ln (1-|b(\tilde{\lambda}, \tilde{\omega})|) d \tilde{\lambda}}
$$


$K$ is a Cauchy-type kernel. $F(\lambda, \omega)$ is a meromorphic function having poles at the upper $\lambda$-half-planes and unit modulus at the real $\lambda$-axes. Let us call zeroes of $a$ and a function $b(\lambda)$ "the spectrum of the rapidly decreasing problem." On being the branch points lying at the real axes the add spectrum should appear which is the nontrivial phase of $a$ at the real $\lambda$-axes.

To solve the inverse scattering problem we must construct a triangular representation of the Jost function if only at the right-hand end.

Theorem 4. The right-hand Jost functions can be presented in a view

$$
\psi_{+}(x, \lambda, \omega)=e^{i \lambda \sigma_{3} x}+\int_{x}^{\infty} \sum_{j=0}^{n-1} K_{j}(x, y) \omega^{j} e^{i \lambda \sigma_{3} y} d y .
$$

The kernels $K_{j}(x, y)$ obey a system of linear partial equation of Goursat type. From their boundary conditions on diagonal $x=y$ one can obtain the formulas connecting $K_{j}(x, y)$ and $\varphi_{i j k}(x)$.

To express the $K_{j}(x, y)$ in terms of a spectrum let us derive the Gel'fandLevitan-Marchenko equations. For that the following relations should be required:

$$
\begin{gathered}
\frac{1}{2 \pi n} \int_{-\infty, \Gamma}^{+\infty} e^{i \lambda x} d \lambda=\delta(x), \\
\frac{1}{2 \pi n} \int_{-\infty, \Gamma}^{-\infty} \omega^{k} e^{i \lambda x} d \lambda=\sum_{i=0}^{k M} a_{i} \delta^{(i)}(x) .
\end{gathered}
$$

When $\varphi_{i j k}(x)$ are purely imaginary

$$
K_{i}(x, y)=\left(\begin{array}{cc}
K_{i}^{1}(x, y) & K_{i}^{2}(x, y) \\
-\bar{K}_{i}^{2}(x, y) & \bar{K}_{i}^{1}(x, y)
\end{array}\right) .
$$

Therefore, one can obtain the Gel'fand-Levitan-Marchenko equation by the same way as in [7]. We obtain

$$
\begin{gathered}
F_{i}(x+y)+\sum_{j=1}^{n-1} \sum_{k=0}^{R_{2}} a_{i j k} \frac{\partial^{k} K_{j}^{2}(x, y)}{\partial y^{k}}+\int_{x}^{\infty} \sum_{j=0}^{n-1} K_{j}^{1}(x, z) F_{i+j}(z+y) d z=0, \\
\sum_{j=0}^{n-1} \sum_{k=0}^{R_{1}} b_{i j k} \frac{\partial^{k} K_{j}^{1}(x, y)}{\partial y^{k}}-\int_{x}^{\infty} \prod_{j=0}^{n-1} \bar{K}_{j}^{2}(x, z) F_{i+j}(z+y) d z=0, \\
F_{i}(x)=-\mathfrak{i} \sum_{k} \frac{b_{k}}{\dot{a}_{k}} e^{i \lambda_{k} x} \omega^{i}-\frac{1}{2 \pi n} \int_{-\infty, \Gamma}^{+\infty} \frac{b(\lambda)}{a(\lambda)} \omega^{i} e^{i \lambda x} d \lambda .
\end{gathered}
$$

These equations make somebody able to restore $\varphi_{i j k}(x)$ from the spectral data of (10).

It should be noted that in definition (10) $\lambda$ may enter also in some finite negative powers. It should not result in any troubles in construction of a triangular representation. But the spectral problem would be more complicated: the additional poles of the transition matrix would appear as it was in the case of solutions of a nonlinear Schrödinger equation with finite density [2].

The following two theorems point out the operators which are researchable by the above mentioned approach. 
Theorem 5. Let $\Gamma$ be a compact Riemann surface, $U(x, \lambda, \omega)$ be a single-valued meromorphic function of $\Gamma$ taking values at the algebra of infinite differentiable $2 \times 2$ matrices with zero trace such that

1. $U \underset{|x| \rightarrow \infty}{\rightarrow} i \lambda \sigma_{3}$.

2. The poles of $U$ coincide with ones of $\lambda$.

Then $U(x, \lambda, \omega)$ may be presented in the form (10).

Theorem 6. Insert the conditions 1 and 2 of Theorem 5 onto

1. $U \underset{|x| \rightarrow \infty}{\rightarrow} U_{0}$.

2. The poles of $U$ coincide with ones of $\sqrt{\operatorname{det} U_{0}}$.

Then, by gauge transformation with constant with respect to $x$ matrix $g, U$ may be transformed to matrix $\widetilde{U}$ such that

1. $\tilde{U} \underset{|x| \rightarrow \infty}{\rightarrow} i \lambda \sigma_{3}$, where $\lambda=\sqrt{\operatorname{det} U_{0}}$.

2. The poles of $\lambda u_{+} u_{-} \tilde{U}$ coincide with ones of $\lambda$.

The matrix $\lambda u_{+} u_{-} \tilde{U}$ obeys the conditions of Theorem 5 with a Riemann surface $\Gamma$ being two sheets covering over $\Gamma$ with branch points lying at zeroes of $\operatorname{det} U_{0}$. Therefore we can construct a triangular representation of Jost functions according to operators obey conditions of Theorem 6 .

\section{Nonlinear Equations Associated with $\operatorname{sl}(2, \mathscr{A})$}

Let $\operatorname{sl}(2, \mathscr{A})$ denote the algebra of $2 \times 2$ matrixes taking $\mathscr{A}$ values in the ring was described in the example of Sect. 1. It includes the algebra generated by

$$
X_{k}=\frac{i \lambda^{k} w_{1} \sigma_{1}}{4}, \quad Y_{k}=\frac{i \lambda^{k} w_{2} \sigma_{2}}{4}, \quad Z_{k}=\frac{i \lambda^{k} w_{1} w_{2} \sigma_{3}}{4} .
$$

$\sigma_{i}-$ are the Pauly matrixes.

Let us divide $\mathfrak{G}(\mathscr{A})$ into subalgebras

$$
\begin{gathered}
\mathfrak{5}\left(\mathscr{A}_{+}\right)=\left\{X_{i}, Y_{i}, Z_{j} \mid i \geqq 1, j \geqq 2\right\}, \\
\mathfrak{5}\left(\mathscr{A}_{-}\right)=\left\{X_{i}, Y_{i}, Z_{i} \mid i \leqq-1\right\},
\end{gathered}
$$

and an addition $\left(\mathfrak{5}\left(\mathscr{A}_{0}\right)=\left\{X_{0}, Y_{0}, Z_{0}, Z_{1}\right\}\right.$. The commutator (1) with (12) results in following relations:

$$
\begin{aligned}
& {\left[X_{i}, Y_{j}\right]_{0}=\left\{\begin{array}{cc}
-\frac{1}{2} Z_{i+j} & i \geqq 1, j \geqq 1 \\
\frac{1}{2} Z_{i+j} & i \leqq-1, j \leqq-1,
\end{array}\right.} \\
& {\left[Z_{i}, X_{j}\right]_{0}=\left\{\begin{array}{cc}
-\frac{1}{2} Y_{i+j}+\frac{\alpha_{1}}{8} Y_{i+j-2} & i \geqq 2, j \geqq 1 \\
\frac{1}{2} Y_{i+j}-\frac{\alpha_{1}}{8} Y_{i+j-2} & i \leqq-1, j \leqq-1,
\end{array}\right.} \\
& {\left[Y_{i}, Z_{j}\right]_{0}=\left\{\begin{array}{cc}
\frac{1}{2} X_{i+j}+\frac{\alpha_{2}}{8} X_{i+j-2} & i \geqq 1, j \geqq 2 \\
\frac{1}{2} X_{i+j}-\frac{\alpha_{2}}{8} X_{i+j-2} & i \leqq-1, j \leqq-1,
\end{array}\right.}
\end{aligned}
$$


0 in other cases. The elements $X_{0}, Y_{0}, Z_{0}$ have been related to $\left(\mathfrak{G}\left(\mathscr{A}_{0}\right)\right.$ because it is only the case when the Poisson-Kirillov bracket corresponding to (13) is reducible to

$$
M \in \mathbb{G}^{*}, \quad M=\left\{X_{-1}^{*}, Y_{-1}^{*}, Z_{-2}^{*}\right\} .
$$

Let us denote as $\widetilde{\mathfrak{G}}(\mathscr{A})^{\wedge}$ a central extension of the algebra of periodic infinite differentiable with respect to $x$ functions taking their values in $\mathscr{5}(\mathscr{A})$. Its central extension is determined by the cocycle (5) with the 1-form

$$
\omega_{0}=\frac{d \lambda}{4 \pi i w_{1} w_{2}} \text {. }
$$

The Poisson-Kirillov brackets on $\left(\tilde{\mathfrak{F}}(\mathscr{A})^{\wedge}\right)^{*}$ are ultralocal and result in the relation just the same as (13). They are also reducible to $M$, so the bracket (6) for $U \in M$

$$
U=\left(\begin{array}{cc}
\frac{\lambda d \lambda}{4 \pi w_{1} w_{2}} & \frac{\operatorname{Im} \psi d \lambda}{4 \pi w_{1}}-\frac{i \operatorname{Re} \psi d \lambda}{4 \pi w_{2}} \\
\frac{\operatorname{Im} \psi d \lambda}{4 \pi w_{1}}+\frac{i \operatorname{Re} \psi d \lambda}{4 \pi w_{2}} & -\frac{\lambda d \lambda}{4 \pi w_{1} w_{2}}
\end{array}\right)
$$

is equivalent to

$$
\{\psi(x), \bar{\psi}(y)\}=i \delta(x-y)
$$

Let us derive the solution of where

$$
L T=0
$$

$$
L=\partial_{x}-\frac{U}{\omega_{0}}=\left(\begin{array}{cc}
i \lambda & w_{1} \operatorname{Re} \psi+i w_{2} \operatorname{Im} \psi \\
-w_{1} \operatorname{Re} \psi+i w_{2} \operatorname{Im} \psi & -i \lambda
\end{array}\right)
$$

in the form

$$
\begin{aligned}
& T(x, \lambda)=(1+W) e^{i \lambda \sigma_{3} x+\sigma_{3}} \int_{x_{0}}^{x} h(x, \lambda) d x, \\
& W=\left(\begin{array}{cc}
0 & W_{+} \\
W_{-} & 0
\end{array}\right), \quad h=\sum_{k=1}^{\infty} \frac{h_{k}^{0}+w_{1} w_{2} h_{k}^{3}}{(i \lambda)^{k}},
\end{aligned}
$$

$h$ obeys the Riccaty equation,

$$
h_{x}-\frac{w_{1} \operatorname{Re} \psi_{x}-i w_{2} \operatorname{Im} \psi_{x}}{w_{1} \operatorname{Re} \psi-i w_{2} \operatorname{Im} \psi} h+h^{2}+|\psi|^{2}-\frac{1}{4 \lambda^{2}}\left(\alpha_{1}(\operatorname{Re} \psi)^{2}+\alpha_{2}(\operatorname{Im} \psi)^{2}=2 i \lambda h .\right.
$$

From the last equation a set of recurrent relations on $h_{k}^{0}$ and $h_{k}^{3}$ can be obtained. From them it follows that

$$
\begin{aligned}
& \mathscr{H}=\oint_{C_{\lambda}} \arccos \left(\frac{1}{2} \operatorname{Sp} T(2 \pi, 0, \lambda)\right) \omega(\lambda) \\
&=\int_{0}^{2 \pi}\left[-\left|\psi_{x}\right|^{2}+|\psi|^{4}+\frac{\alpha_{1}+\alpha_{2}}{2}|\psi|^{2}+\frac{\alpha_{1}-\alpha_{2}}{4}\left(\psi^{2}+\bar{\psi}^{2}\right)\right] d x \\
& \omega(\lambda)=\frac{\lambda^{2}}{\pi} \mathrm{d} \lambda .
\end{aligned}
$$


The hamiltonian (15) and the bracket (14) result in the following equation

$$
-i \psi_{t}=\psi_{x x}+2|\psi|^{2} \psi+\frac{\alpha_{1}+\alpha_{2}}{2} \psi+\frac{\alpha_{1}-\alpha_{2}}{2} \bar{\psi},
$$

which is the perturbed nonlinear Schrödinger equation. Its representation may be derived from (7) as

$$
U_{t}=\{U, \mathscr{H}\}=\left[A_{1}, U\right]+A_{1 x} \omega_{0}-\hat{P}_{0}^{*}\left(\left[A_{1}, U\right]+A_{1 x} \omega_{0}\right)+\hat{P}_{-}^{*}\left(\left[A_{2}, U\right]+A_{2 x} \omega_{0}\right),
$$

where

$$
A_{1}=\hat{P}_{+}\left(\frac{i \omega}{2 \omega_{0}} M\right), \quad A_{2}=\hat{P}_{0}\left(\frac{i \omega}{2 \omega_{0}} M\right) .
$$

$\hat{P}_{+}$and $\hat{P}_{0}$ are defined by the kernels (4). Matrix $M$ is a solution of the equation

$$
[M, U]+M_{x} \omega_{0}=0 .
$$

Let us derive it in the form

$$
\begin{aligned}
& M=\left(\begin{array}{cc}
f & g \\
\tilde{g} & -f
\end{array}\right), \quad f=1+\sum_{k=1}^{\infty} \frac{f_{k}^{0}+w_{1} w_{2} f_{k}^{3}}{\lambda^{k}}, \\
& g=\sum_{k=1}^{\infty} \frac{w_{1} g_{k}^{1}+w_{2} g_{k}^{2}}{\lambda^{k}}, \quad \tilde{g}=\sum_{k=1}^{\infty} \frac{w_{1} \tilde{g}_{k}^{1}+w_{2} \tilde{g}_{k}^{2}}{\lambda^{k}},
\end{aligned}
$$

thus, we shall also find a set of recurrent relations on $g_{k}^{1}, g_{k}^{2}, \tilde{g}_{k}^{1}, \tilde{g}_{k}^{2}, f_{k}^{0}, f_{k}^{3}$. Computing coefficients attached to the lowest two powers one can obtain the final representation

with

$$
L_{t}=[L A]+\varepsilon B
$$

$$
\begin{gathered}
A=\left(\begin{array}{cc}
-2 w_{1} w_{2} \lambda^{2}+|\psi|^{2} w_{1} w_{2} & \\
-2 w_{1} \lambda \operatorname{Im} \psi-2 i w_{2} \lambda \operatorname{Re} \psi+w_{2} \operatorname{Re} \psi_{x}-i w_{1} \operatorname{Im} \psi_{x} & 2 i w_{2} \lambda \operatorname{Re} \psi-2 w_{1} \lambda \operatorname{Im} \psi+w_{2} \operatorname{Re} w_{x}+i w_{1} \operatorname{Im} \psi_{x} \\
2 w_{1} w_{2} \lambda^{2}-|\psi|^{2} w_{1} w_{2}
\end{array}\right), \\
\varepsilon B=\left(\begin{array}{cc}
\frac{\alpha_{1}-\alpha_{2}}{2 \lambda} \operatorname{Re} \psi \operatorname{Im} \psi & -\frac{w_{2} \alpha_{1}}{2 \lambda^{2}}|\psi|^{2} \operatorname{Re} \psi-\frac{i w_{1} \alpha_{2}}{2 \lambda^{2}}|\psi|^{2} \operatorname{Im} \psi \\
-\frac{w_{2} \alpha_{1}}{2 \lambda^{2}}|\psi|^{2} \operatorname{Re} \psi+\frac{i \alpha_{2} w_{1}}{2 \lambda^{2}}|\psi|^{2} \operatorname{Im} \psi & -\frac{\alpha_{1}-\alpha_{2}}{2 \lambda} \operatorname{Re} \psi \operatorname{Im} \psi
\end{array}\right) .
\end{gathered}
$$

The Poisson bracket of the transition coefficients is given by (9).

The approach mentioned above can be also applied to the equation

$$
\psi_{t}=\psi_{x x x}+2|\psi|^{2} \psi_{x}+\frac{\beta_{1}+\beta_{2}}{2} \psi+\frac{\beta_{1}-\beta_{2}}{2} \bar{\psi},
$$


in that case

$$
\begin{aligned}
& w_{1}^{2}=1-\frac{\alpha}{4 \lambda^{2}}-\frac{\beta_{1}}{8 i \lambda^{3}}, \quad w_{2}^{2}=1-\frac{\alpha}{4 \lambda^{2}}-\frac{\beta_{2}}{8 i \lambda^{3}}, \\
& L=\partial_{x}-\left(\begin{array}{cc}
i \lambda & w_{1} \operatorname{Re} \psi+i w_{2} \operatorname{Im} \psi \\
-w_{1} \operatorname{Re} \psi+i w_{2} \operatorname{Im} \psi & -i \lambda
\end{array}\right) \text {, } \\
& \omega_{0}=\frac{d \lambda}{4 \pi i w_{1} w_{2}}, \\
& \mathscr{H}=\oint_{C_{\lambda}} \arccos \left(\frac{1}{2} \operatorname{Sp} T(2 \pi, 0, \lambda)\right) \omega(\lambda) \\
& =\int_{0}^{2 \pi}\left[\frac{\bar{\psi}_{x x} \psi_{x}-\bar{\psi}_{x} \psi_{x x}}{2 i}+|\psi|^{2} \frac{\bar{\psi} \psi_{x}-\bar{\psi}_{x} \psi}{2 i}\right. \\
& \left.+\frac{\beta_{1}+\beta_{2}}{2}|\psi|^{2}+\frac{\beta_{1}-\beta_{2}}{4}\left(\psi^{2}+\bar{\psi}^{2}\right)\right] d x \\
& \omega(\lambda)=\left(1-\frac{i}{w_{1} w_{2}}\right) \frac{2 \lambda^{3} d \lambda}{\pi} \\
& P_{0}(\tilde{\lambda}, \lambda)=\left(1+\left(\frac{\tilde{w}_{1}}{w_{1}}+\frac{\tilde{w}_{2}}{w_{2}}+\frac{\tilde{w}_{1} \tilde{w}_{2}}{w_{1} w_{2}}\right)\left(1+\frac{\tilde{\lambda}}{\lambda}\right)\right. \\
& \left.+\frac{\tilde{w}_{1} \tilde{w}_{2}}{w_{1} w_{2}} \frac{\tilde{\lambda}^{2}}{\lambda^{2}}\right) \frac{d \lambda}{8 \pi i \lambda} \\
& P_{-}(\tilde{\lambda}, \lambda)=\left(\frac{d \lambda}{16} \delta(\tilde{\lambda}-\lambda)+\text { V.p. } \frac{d \lambda}{8 \pi i(\lambda-\tilde{\lambda})}\right)\left(1+\frac{\tilde{w}_{1}}{w_{1}}+\frac{\tilde{w}_{2}}{w_{2}}+\frac{\tilde{w}_{1} \tilde{w}_{2}}{w_{1} w_{2}}\right) \text {, } \\
& P_{+}(\tilde{\lambda}, \lambda)=\delta_{c}(\tilde{\lambda}, \lambda)-P_{0}(\lambda, \tilde{\lambda})-P_{-}(\tilde{\lambda}, \lambda),
\end{aligned}
$$

and also for the system

with

$$
\begin{gathered}
-i u_{t}=u_{x x}+16 u|u|^{2}+a v \\
-i v_{t}=v_{x x}+16 v|v|^{2}+\alpha u
\end{gathered}
$$

$$
\begin{gathered}
w_{1}^{2}=1-\frac{\alpha}{4 \lambda^{2}}, \quad w_{2}^{2}=1+\frac{\alpha}{4 \lambda^{2}}, \\
L=\partial_{x}-\left(\begin{array}{cc}
i \lambda w_{1} w_{2} & \sqrt{2} w_{1}(u+v)+\sqrt{2} w_{2}(u-v) \\
-\sqrt{2} w_{1}(\bar{u}+\bar{v})+w_{2}(\bar{v}-\bar{u}) \sqrt{2} & -i \lambda w_{1} w_{2}
\end{array}\right), \\
\mathscr{H}=\oint_{C_{\lambda}} \arccos \left(\frac{1}{2} \operatorname{Sp} T(2 \pi, 0, \lambda)\right) \omega(\lambda) \\
=\int_{0}^{2 \pi}\left[-\left|u_{x}\right|^{2}+8|u|^{4}+\alpha(u \bar{v}+\bar{u} v)-\left|v_{x}\right|^{2}+8|v|^{4}\right] d x \\
\omega=\frac{\lambda^{2} d \lambda}{4 \pi w_{1} w_{2}}, \quad \omega_{0}=\frac{d \lambda}{8 \pi i w_{1} w_{2}}, \\
\{u(x), \bar{u}(y)\}=i \delta(x-y), \quad\{v(x), \bar{v}(y)\}=i \delta(x-y) .
\end{gathered}
$$

The kernels $P_{ \pm}(\tilde{\lambda}, \lambda)$ and $P_{0}(\tilde{\lambda}, \lambda)$ are defined by (4).

One can directly verify that when $\alpha=O(\varepsilon)$ and $\beta=O(\varepsilon)$ then all the above equations admit the representation $\left(7^{\prime}\right)$. 


\section{Conclusion}

The inverse scattering problem (ISP) is a good technique to investigate solutions of near-integrable equations [8]. On a small perturbation the solution should also be a nonlinear sum of solitons with parameters being slowly changed and continuous spectrum excitations. If there are no excitations in continuous spectrum in a moment, one can ignore its influence on solitions at the first order. On some additional conditions the amplitude of excitations of continuous spectrum would be small for all the time. It is a consequence of the property of the most integrable systems: on a small perturbation the invariance of the most invariant tori is conserved.

In a paper [9] a simple perturbation technique was developed which uses the ISP as a canonical transform of a phase space of rapidly decreasing functions. It results in one being able to derive the first order of evolution of the solitonic parameters and excitations in continuous spectrum without any manipulations with Jost functions. The transform of the phase space is a transition to the expressions of spectral data to have canonical Poisson brackets.

In that paper only the case of hamiltonian perturbation theory with perturbed hamiltonian and unperturbed Poisson brackets was considered. As it follows from (9), the equations of Sect. 4 provide the opposite example of hamiltonian perturbation theory: after transition to spectral data of new $L$-operator one should obtain a hamiltonian system for which hamiltonians have the same structure but the Poisson bracket is perturbed. So the perturbation

$$
\varepsilon \mathscr{H}_{a}=\int\left[\frac{\alpha_{1}+\alpha_{2}}{2}|\psi|^{2}+\frac{\alpha_{1}-\alpha_{2}}{4}\left(\psi^{2}+\bar{\psi}^{2}\right)\right] d x
$$

of the nonlinear Schrödinger equation may be described only by deformation of the Poisson brackets.

The induction of a new $L$-operator for (16) has the following reason: it glues by small hands lying near zeroes of $\lambda$ two Riemann surfaces which correspond to finite-gap solutions being different from one another only by $\psi \rightarrow \bar{\psi}$. On being zeroes of Baker-Akhiezer function which move from one piece of the new Riemann surface to another we shall say: "there is a bifurcation of invariant torus". So the perturbation (17) may result not only in slow deformations of invariant tori but also in their bifurcations. In particular, in the rapidly decreasing case solitons of a new type may appear, perhaps because the perturbation (17) takes off the phase degeneracy of the nonlinear Schrödinger equation.

The statement is similar to the anisotropic Landau-Lifshitz equation,

$$
\mathbf{S}_{t}=\left[\mathbf{S}, \mathbf{S}_{x x}\right]+[\mathbf{S}, \mathscr{T} \mathbf{S}], \quad \mathscr{T}=\operatorname{diag}(-4 \alpha,-4 \beta, 0) .
$$

On any nonvanished $\alpha$ and $\beta$ it has a solution of domain wall type. It has no such solutions when $\alpha=0, \beta=0$ and they could not be obtained in the perturbation framework with respect to $\alpha$ and $\beta$. The reason is just the same: after transition from $\alpha=0, \beta=0$ to $\alpha \neq 0, \beta \neq 0$ the axial and polar degeneracies would be taken off. In that example the integrability is kept after the deformations, but it is not so in most of the other cases.

The inverse scattering problem for the $L$-operators associated with the equations which have been considered in Sect. 4 may be solved as well as in the rational case. To restore the potentials from scattering data one must use the 
techniques of Sect. 3. The evolution of scattering data due to the nonlinear system is defined by Eq. (7). Since a potential is reflectionless at the initial moment, $r=0$, it should be $r=O(\varepsilon)$ for a finite time (in the resonantless case it is true for all time), here $r$ is a reflection coefficient. It results in our being able to reduce our dynamic system to a submanifold of reflectionless potentials at the first order of $\varepsilon$. It should be emphasized that the equations for which those potentials are exact solutions either do not exist or have much more complicated nonlocal form.

The hamiltonian (15) or similar may also be perturbed by terms which do not change symmetries. One may consider their influence on solitions by the techniques of [9], preliminarily calculating the Poisson brackets of scattering data using (9). The last term in (9) can be omitted at first order.

More detailed calculations according to some interesting case from the physicist's point of view will be presented elsewhere in my publications.

Acknowledgements. I am very thankful to Prof. B. A. Dubrovin, Prof. I. M. Krichever, and Prof. S. P. Novikov for many discussions of this paper.

\section{References}

1. Kostant, B.: The solution to a generalization Toda lattice and representation theory. Adv. Math. 34, 195-338 (1979)

2. Takhtajan, L.A., Faddeev, L.D.: Hamiltonian approach in soliton theory. Berlin, Heidelberg, New York: Springer 1985

3. Mikhalev, V.G.: Complete integrability of the asymmetric chiral $O(3)$-field equation in a class of rapidly decreasing functions. Physica D 40, 421-432 (1989)

4. Krichever, I.M., Novikov, S.P.: The algebras of Virasoro type, Riemann surfaces and structures of soliton theory. Funkz. Analiz. 21, 46-63 (1987)

5. Semenov-Tyan-Shansky, M.A.: What is a classical $r$-matrix? Funkz. Analiz. 17, 17-33 (1983)

6. Zakharov, V.E., Mikhaylov, A.V.: Funkz. Analiz. 17, 1-10 (1983)

7. Zakharov, V.E., Manakov, S.V., Novikov, S.P., Pitaevski, L.P.: Theory of solitons. Moscow: Nauka 1980

8. Karpman, V., Maslov, E.: The soliton perturbation theory. Sov. J. Phys. (ZETP) 73, 538-559 (1977)

Kaup, D., Newell, A.: Solitons as particles and oscillators. Proc. R. Soc. London $361 \mathrm{~A}$, 413-446 (1978)

McLaughlin, D., Scott, A.: Appl. Phys. Lett. 30, 545 (1978)

9. Mikhalev, V.G.: The investigation of the nonlinear one-dimensional systems by hamiltonian formalism. Teor. Mat. Fiz. 76, 199-206 (1988) 